\title{
PERSPECTIVES
}

\section{Refinement in Asian blepharoplasty for the aging}

\section{Apirag Chuangsuwanich}

Division of Plastic Surgery, Department of Surgery, Faculty of Medicine Siriraj Hospital, Mahidol University, Bangkok, Thailand

\begin{abstract}
Signs of an aging face beginning at the periorbital region are easily noticeable and may require surgical correction. The hanging of upper eyelids, tired look, and sunken upper eyelids are common symptoms and signs. Most of the described techniques of blepharoplasty for the aging emphasize on the excision of the hanging eyelid skin which will leave patients with long scar. However, eyebrow and eyelid ptosis are common among aging people. Therefore, undertaking only an upper blepharoplasty does not necessarily yield good aesthetic results. This paper describes a modified blepharoplasty technique with limited incision. The operation also includes eyebrow reposition and/or correction of eyelid ptosis that further improve results. An asymmetry of the frontalis muscle could cause asymmetry in the eyelid folds post-operatively. However, botulinum toxin injection may correct this problem.
\end{abstract}

Keywords: blepharoplasty; blepharoptosis; eyelids

Citation: Chuangsuwanich A. Refinement in Asian blepharoplasty for the aging. J Surg Dermatol 2021; 6(1): 14; http://dx.doi.org/10.18282/jsd.v6.i1.14.

*Correspondence to: Apirag Chuangsuwanich, Division of Plastic Surgery, Department of Surgery, Faculty of Medicine Siriraj Hospital, Mahidol University, Bangkok, Thailand; apirag@gmail.com

Received: $12^{\text {th }}$ August 2020; Accepted: $28^{\text {th }}$ October 2020; Published Online: $8^{\text {th }}$ November 2020

\section{Overview}

Signs of aging usually begin at the periorbital area. The hanging of upper eyelids, tired look and sunken upper eyelids are common symptoms and signs. Most of the described techniques of blepharoplasty for the aging emphasize on the excision of the hanging eyelid skin which will leave patients with long scar ${ }^{[1-3]}$. The results will look unnatural alongside lagophthalmos and will also leave a noticeable $\operatorname{scar}^{[4,5]}$. Eyelid ptosis is also a common finding among the aging. If this problem is not corrected, the results of the operation will be compromised. In the author's opinion, the hanging of the eyelid might not only be caused by excess skin but also by brow ptosis. The eyelid fold asymmetry could be avoided by correcting the eyelid ptosis ${ }^{[6]}$. Good physical examinations for the signs of aging in each patient will determine the techniques used for correction. This article presents a refined technique for Asian blepharoplasty using limited incision blepharoplasty with the correction of brow position and eyelid ptosis, and also the use of botulinum toxin in correcting eyelid crease asymmetry.

\section{Ideas and innovation}

The short incision blepharoplasty has been described for oriental eyelids previously by the same author in the article "Short incisional double eyelid blepharoplasty for Asian people"[7]. In this article, two relevant findings were emphasized: firstly, the orbital septum is quite thick, and secondly, there is a tiny transverse band of fascia running across the medial canthal area to the lateral orbital wall at the superior border of the tarsal plate. The author called this band the "pre-tarsal tendon". Here, the aforementioned blepharoplasty procedure is modified for the correction of aging eyelids. The method can also include eyebrow reposition and ptosis correction. The sequences of the corrections are as follows:

Copyright (C) 2021 Chuangsuwanich A. This is an Open Access article distributed under the terms of the Creative Commons Attribu-tionNonCommercial 4.0 International License (http://creativecommons.org/licenses/by-nc/4.0/), permitting all non-commercial use, distribution, and reproduction in any medium, provided the original work is properly cited. 


\section{Pre-operative evaluation}

\section{Patient history and expectation}

History of previous operation or trauma around the orbit, allergy, and any drug and herbal medicine used should be noted. Foreign material injection on the face is not uncommon in Asians and the material injected could migrate to the periorbital area, causing eyelid swelling. The patient's expectations and motivation of the surgery should also be carefully considered. If these are not reasonable, the operation should not be done ${ }^{[8,9]}$. Usually the author will simulate the new eyelid crease to the patients in front of a mirror and inquire their likes or dislikes.

\section{Physical examination}

The detail and symmetry of the eye's globe position, lid creases and fullness, bony orbit, eyebrow position, and lid opening including levator function should be thoroughly examined and recorded. In order to determine whether the eyelid skin should be excised or not, the author will position the patient's brow to the superior orbital rim while the patient is in an upright position, and then create an eyelid crease with a blunt-point stick. If the created eyelid fold is suitable, the eyelid skin will not be excised. Many patients have asymmetry of eyelid creases and openings because of unequal eyebrow position or eyelid ptosis ${ }^{[10]}$. Asymmetry of frontalis function should also be observed. On the hyper-activity site, additional skin creases at the forehead may be noticed. Photographs for planning the procedure and keeping a record are essential. The photos of front, lateral and bird's eye view should be taken.

\section{Indication for surgery}

Patients who have the hooding of upper eyelid skin, as well as patients with low, lost or asymmetric supratarsal folds with eyebrows lower than superior orbital rims, are candidates for the following described procedures. Asymmetry of the eyebrows can affect the lid crease $^{[6,11]}$. The brows should be above the superior orbital rim in women and at the rim in men ${ }^{[12-14]}$. Low eyebrows can cause lid hooding that leads to sad expression.

\section{Patient selection criteria}

Patient selection criteria are, firstly, patients who have hanging upper eyelid skin over the supratarsal fold with an eyebrow position lower than the superior orbital rim; and secondly, patients who have asymmetry of the eyebrow position and asymmetry of the supratarsal fold.

\section{Surgical technique}

\section{Limited incision blepharoplasty with eyebrow reposition $^{[13]}$ (Figure 1)}

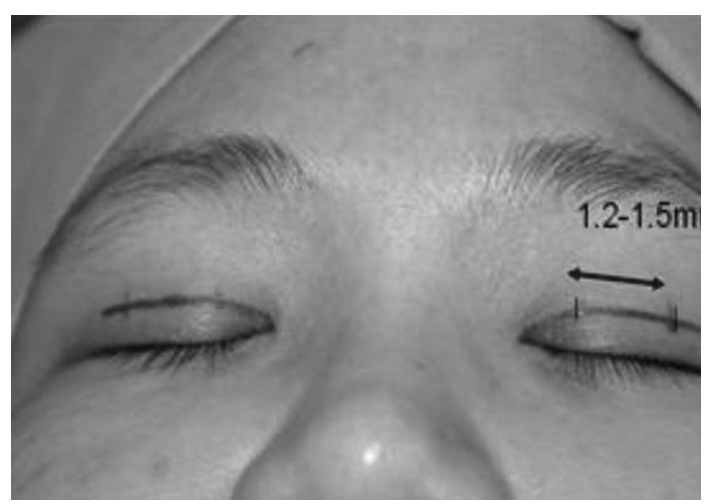

Figure 1. Limited incision blepharoplasty. A point was marked at 6 to $8 \mathrm{~mm}$ above the eyelash on the central portion of the eyelid. The medial end of the line of incision started close to the lid margin. The line curved to central marked point and the lateral end of the mark remained at the same height as the central part, finishing at medial to lateral orbital rim.

Under sterile condition, a line was marked at 6 to $8 \mathrm{~mm}$ position above the eyelash in the central portion of the eyelid and was extended to the medial and lateral parts of the upper eyelid. The medial end of this mark should come close to the lid margin. The lateral end of the mark remained at the same height as the central part. The line of the incision was drawn from above the medial canthal area to just medial to the lateral orbital rim. After repositioning the eyebrow to a proper level, the excess eyelid skin may be excised if appropriate. However, the incision should not pass beyond the lateral orbital rim. Lidocaine with adrenaline (1\%, $4 \mathrm{ml})$ was injected subcutaneously. The skin was cut with a No. 15 blade. The orbicuralis muscle and orbital septum were cut using bipolar cautery. The orbicuralis muscle beneath the incision was excised 1 or $2 \mathrm{~mm}$ only, but the muscle under the lateral orbital rim should be cut completely for the lifting of the eyebrow. Pre-tarsal tendon should be removed if it was present (Figure 2).

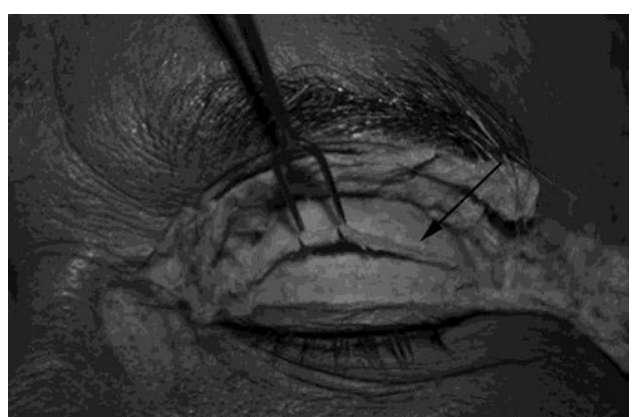

Figure 2. The pre-tarsal tendon is marked with black arrow 
The central fat pad was also removed. The superior skin flap was dissected and retracted to expose the periosteum of the superior orbital rim. The 4-0 nylon was used to fix the dermis of the lateral eyebrow to be $1 \mathrm{~cm}$ above the superior orbital rim (Figure 3). One more fix to the eyebrow at the medial limbus area would be added if necessary. The eyebrow correction was done in $78 \%$ of the patients except in young patients without excess skin. The patients had a dimple under the brow, which would fade out within one month. Two modification stitches of the dermis of the lower incision edge to the upper border of tarsal plate were performed using clear 6-0 undyed nylon at sites perpendicular to the pupil and lateral limbus of the corneal level. Hemostasis was then completed. The incision was closed with 6-0 undyed nylon using subcuticular interrupted technique. Topical eye ointment was applied over the wounds without taping. Cold compression should be applied immediately post-operative and continued for 24 hours. Prophylactic antibiotic for pyogenic cocci and analgesic drug were prescribed for five days. Four days after operations, patients could wash their faces.

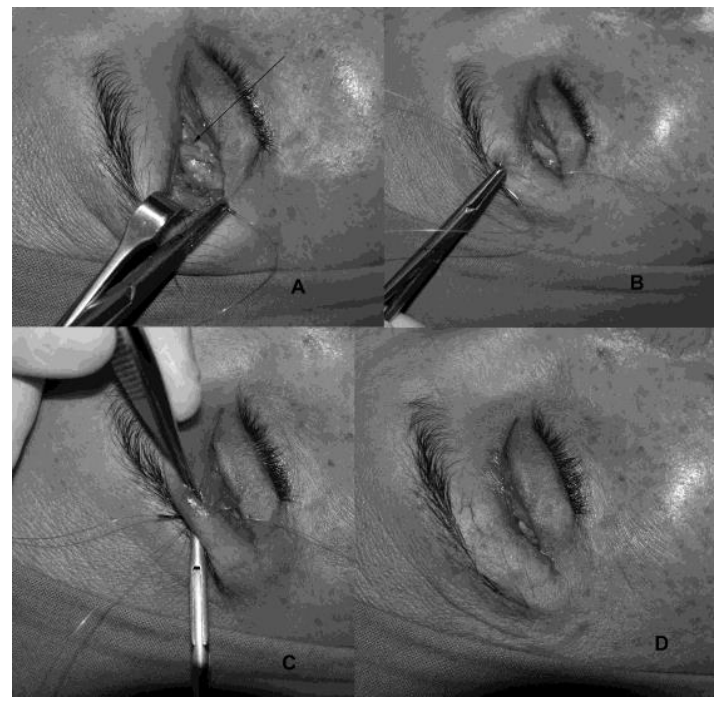

Figure 3. Brow reposition done through blepharoplasty incision. (A) 4-0 nylon suture at $1 \mathrm{~cm}$ above superior orbital rim. The arrow points at the superior orbital rim (B) The needle was passed through the skin at the lower border of the brow (C) The needle was passed through the same hole (D) After tying the suture, the brow was lifted up

\section{Limited incision blepharoplasty in eyelid ptosis patient}

Eyelid ptosis were graded as below ${ }^{[4]}$ :

1. Mild ptosis: lid covers limbus $1-2 \mathrm{~mm}$

2. Moderate ptosis: lid covers limbus $3-4 \mathrm{~mm}$

3. Severe ptosis: lid covers limbus $>4 \mathrm{~mm}$
Before the operation, the surgeon should evaluate for the excursion of levator superioris. Levator function is graded as poor (excursion $=0-4 \mathrm{~mm})$, moderate $(5-10$ $\mathrm{mm})$ and good $(>10 \mathrm{~mm})$. Ptosis should be corrected at the same time when the lid crease surgery is performed; otherwise, the symmetry of lid crease is unable to be achieved especially in the unilateral ptosis. The ptosis correction described in most references used long upper eyelid incision $(>20 \mathrm{~mm})^{[14,15]}$. In this technique, the correction was able to be performed with a shorter incision $(10-15 \mathrm{~mm})$. The steps of the operation were the same as the steps described above. Using the keyhole technique, the levator expansion and tarsal plate could be exposed from the medial to the lateral border. In mild-to-moderate ptosis with moderate-to-good levator function, levator plication with 5-0 nylon and with horizontal mattress technique was done at the pupil and lateral limbus point (Figure 4). The distance of plication should be a $1: 2$ or 1:3 ratio, according to the lid opening ${ }^{[4,5]}$. The symmetry should be checked at both the lid crease and the eye opening before wound closure.

\section{Botulinum toxin in blepharoplasty}

One of the causes of eyebrow asymmetry is the imbalance of the frontalis muscle activity ${ }^{[11,12]}$. The unequal glabella crease and prominent upper border of the eyebrow are signs of frontalis activity. This imbalance in activity can complicate blepharoplasty for patients with unequal eyelid folds that cannot be corrected surgically. In this situation, the author would use botulinum toxin injection on the hyper-activity site at the upper part of frontalis (at the hair line level) at two points and $1 \mathrm{~cm}$ $\operatorname{apart}^{[15]}$ (Figure 5).

The injection would reduce the activity of the frontalis and lower the eyebrow. The patient may need one or two more injections at an interval of three to four months.

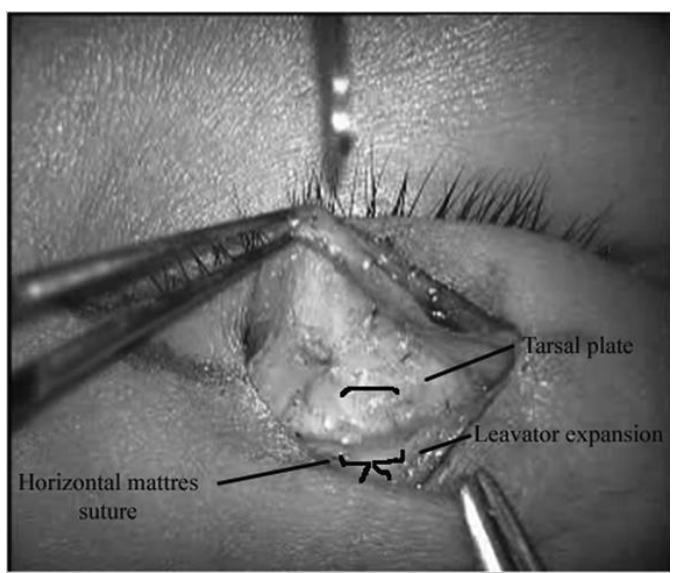

Figure 4. Levator plication with 5-0 nylon using horizontal mattress technique was done at the pupil and lateral limbus point 


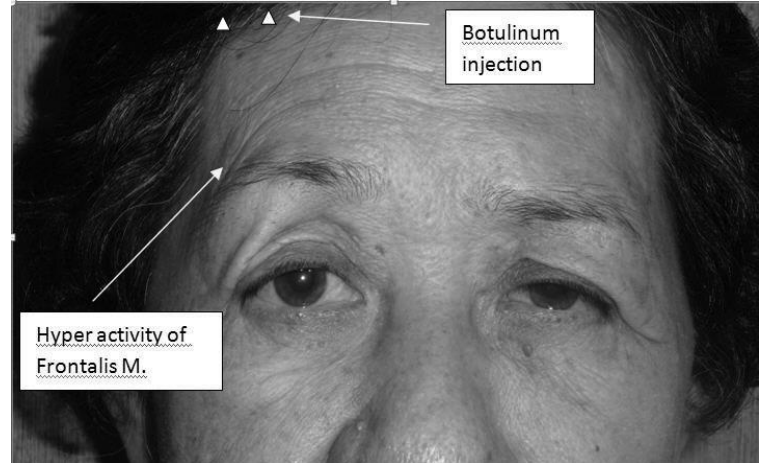

Figure 5. Botulinum injection: This patient had hyper activity of frontalis muscle on the right brow. The marks locate the injection sites.

\section{Results}

These sequences for blepharoplasty for the aging have been applied by the author since 2007 . Between January 2007 to December 2015, 242 patients of age 46-68 years old were operated for aging upper eyelid with brow ptosis, using limited incision blepharoplasty and browpexy. The browpexy was done in 188 patients (75\%). 85 patients $(35 \%)$ needed the ptosis corrections. Follow-up periods were between four months to one year. The botulinum injections were done in 27 patients. $91 \%$ of the patients $(221 / 242)$ were satisfied with the results. Two patients were demonstrated. (Figures 6 and 7) The common consequence was a dimple at the sutured area, which faded out after one month. Unequal eyelid folds occurred in 15 patients $(6 \%)$ that were corrected by re-operations. No serious complication occurred.

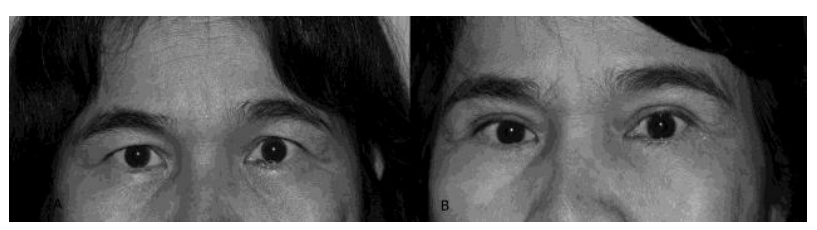

Figure 6. A 55-year-old female with brow ptosis: (A) Pre-operative (B) 6-month post-operation

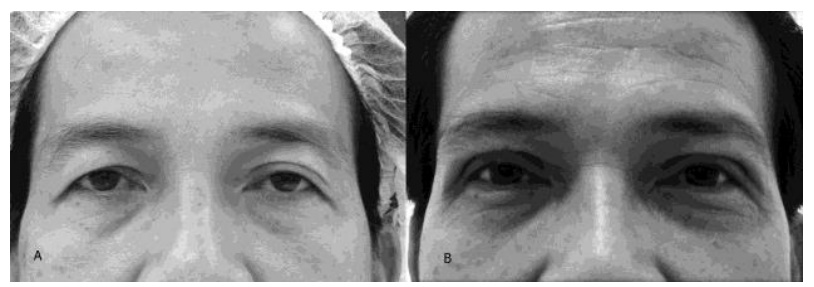

Figure 7. A 56-year-old male with asymmetry brow ptosis: (A) Pre-operative (B) 4-month post-operative

\section{Discussion}

Upper blepharoplasty for the aging is a common operation. Patients usually come with complaints of hanging eyelid skin, loss of eyelid folds, and a tired or sad look. The operations described for the correction of these problems emphasized on the excision of the upper eyelid skin. However, the results would not look natural. Thorough consultation for patients' liking, physical examination and good surgical technique are the keys to reach the goal. If the brow is not in a good position, the surgical technique should correct its causes with minimal scarring because scars that extend through the lateral orbital rim could be as noticeable as crow's feet.

Limited incision blepharoplasty described above will be able to correct these causes with minimal scar and recovery period. Furthermore, the technique can address other causes that might interfere with the results at the same time, such as eyelid ptosis and eyebrow position. Levator plication or advancement can correct ptosis with fair-to-moderate levator function. If the patient has poor levator function, frontalis transfer is the appropriate technique. The limited incision blepharoplasty technique should be one of the choices for Asian blepharoplasty. Frontalis action should also be considered because some patients have the habit of raising only one side of their brow unintentionally ${ }^{[13-15]}$. This will cause unequal eyelid creases that cannot to be corrected surgically. Botulinum toxin is a good option for resolving this problem.

\section{References}

1. Yoon KC, Park SP. Systematic approach and selective tissue removal in blepharoplasty for young Asians. Plast Reconstr Surg 1998; 102(2): 502-508. doi:10.1097/ 00006534-199808000-00035.

2. Chen WPD, Park JDJ. Asian upper lid blepharoplasty: An update on indications and technique. Facial Plast Surg 2013; 29(1): 26-31. doi: 10.1055/s-0033-1333832.

3. Trussler AP, Rohrich RJ. MOC-PSSM CME Article: Blepharoplasty. Plast Reconstr Surg 2008; 121(3): 1-10. doi: 10.1097/01.prs.0000294667.93660.8b.

4. Finsterer J. Ptosis: Causes, presentation, and management. Aesth Plast Surg 2003; 27(3): 193-204. doi: 10.1007/ s00266-003-0127-5.

5. Jung Y, La TY. Blepharoptosis repair through the small orbital septum incision and minimal dissection technique in patients with coexisting dermatochalasis. Korean $\mathbf{J}$ Ophthalmol 2013; 27(1): 1-6. doi: 10.3341/kjo.2013. 27.1.1. 
6. Chen SHT, Mardini S, Chen HC, Chen LMJ, Cheng MH, et al. Strategies for a successful corrective Asian blepharoplasty after previously failed revisions. Plast Reconstr Surg 2004; 114(5): 1270-1277. doi: 10.1097/01.PRS. 0000135951.55118.59.

7. Chuangsuwanich A. Short incisional double eyelid blepharoplasty for Asian people. Aesthetic Surg J 2006; 26(3): 280-286. doi: 10.1016/j.asj.2006.03.008.

8. McCurdy JA. Beautiful eyes: Characteristics and application to aesthetic surgery. Facial Plast Surg 2006; 22(3): 204-214. doi: 10.1055/s-2006-950179.

9. Trussler AP, Rohrich RJ. MOC-PS(SM) CME Article: Blepharoplasty. Plast Reconstr Surg 2008; 121(1S): 1-10. doi: 10.1097/01.prs.0000294667.93660.8b.

10. Jelks GW, Jelks EB. Preoperative evaluation of the blepharoplasty patient: Bypassing the pitfalls. Clin Plast Surg 1993; 20(2): 213-223.
11. Codner MA, Kikkawa DO, Korn BS, Pacella SJ. Blepharoplasty and brow lift. Plast Reconstr Surg 2010; 126(1): 1e-17e. doi: 10.1097/PRS.0b013e3181dbc4a2.

12. Tyers AG. Brow lift via the direct and trans-blepharoplasty approaches. Orbit 2006; 25(4): 261-265. doi: 10. 1080/01676830600977384.

13. McCord CD, Doxanas MT. Browplasty and browpexy: An adjunct to blepharoplasty. Plast Reconstr Surg 1990; 86(2): 248-254. doi: 10.1097/00006534-199008000-00007.

14. Bellinvia G, Klinger F, Maione L, Bellinvia P. Upper lid blepharoplasty, eyebrow ptosis, and lateral hooding. Aesthet Surg J 2013; 33(1): 24-30. doi: 10.1177/10908$20 X 12468751$.

15. Ahn KY, Park MY, Park DH, Han DG. Botulinum toxin A for the treatment of facial hyperkinetic wrinkle lines in Koreans. Plast Reconstr Surg 2000; 105(2): 778-784. doi: 10.1097/00006534-200002000-00050. 